\title{
A Comparative Study on Insulin Secretion, Insulin Resistance and Thyroid Function in Patients with Polycystic Ovary Syndrome with and without Hashimoto's Thyroiditis
}

\author{
Hongli Zhao',* \\ Ying Zhang ${ }^{2, *}$ \\ Jianhong $\mathrm{Ye}^{3}$ \\ Hongjiang $\mathrm{Wei}^{4}$ \\ Zhengping Huang ${ }^{5}$ \\ Xiaoyan Ning ${ }^{6}$ \\ Xiaoying $\mathrm{Fu}^{\prime}$ \\ 'Department of Endocrinology, \\ Guangdong Second Provincial General \\ Hospital, Guangzhou, Guangdong, \\ 510317, People's Republic of China; \\ ${ }^{2}$ Department of Endocrinology, The \\ Third Affiliated Hospital of Guangzhou \\ Medical University, Guangzhou, 5I0I50, \\ People's Republic of China; ${ }^{3}$ Department \\ of Endocrinology, Foshan Hospital of \\ Traditional Chinese Medicine, Foshan, \\ 528000, People's Republic of China; \\ ${ }^{4}$ Outpatient Department, Guangdong \\ Second Provincial General Hospital, \\ Guangzhou, 510317, People's Republic of \\ China; ${ }^{5}$ Department of Rheumatology \\ and Immunology, Guangdong Second \\ Provincial General Hospital, Guangzhou, \\ 5I03 I7, People's Republic of China; \\ ${ }^{6}$ Department of Gastroenterology, \\ Guangdong Second Provincial General \\ Hospital, Guangzhou, 510317, People's \\ Republic of China
}

*These authors contributed equally to this work

Correspondence: Jianhong Ye Department of Endocrinology, Foshan Hospital of Traditional Chinese Medicine, No. 6, Qinren Road, Chancheng District, Foshan, Guangdong Province, 528000,

People's Republic of China

Tel/Fax +86 0757-8306610 I

Email jhy_ye@outlook.com
Objective: The incidence of Hashimoto's thyroiditis (HT) in patients with polycystic ovary syndrome (PCOS) is significantly higher than in normal controls, and there is a risk of more severe metabolic symptoms when the two diseases occur together. This study compares insulin secretion, insulin resistance (IR) and thyroid function in patients with PCOS with and without HT.

Methods: A total of 164 patients (52 patients with HT (HT+) and 112 patients without HT diagnosed PCOS at our hospital were enrolled for testing of oral glucose tolerance, insulin release, thyroid function, the presence of thyroglobulin and thyroid peroxidase antibodies, and blood lipid levels.

Results: Patients with PCOS and HT had higher insulin secretion and IR levels than those without HT, while free thyroxine and thyrotropin levels were significantly lower. The ratio of free thyroxine to thyrotropin was higher in patients with HT.

Conclusion: HT may related with IR and relatively low thyroid function in patients with PCOS. Thus, thyroid function and autoimmune status in patients with PCOS should be evaluated in clinical practice.

Keywords: polycystic ovary syndrome, Hashimoto's thyroiditis, insulin resistance, oral glucose tolerance test

\section{Introduction}

Polycystic ovary syndrome (PCOS) is a highly prevalent disorder, representing the single most common endocrine-metabolic disorder in reproductive-aged women and is characterized by ovulatory dysfunction and/or androgen excess or polycystic ovaries. ${ }^{1}$ PCOS is found in $6-10 \%$ of women of childbearing age. ${ }^{2,3}$ Thyroid dysfunction can affect the female reproductive and endocrine systems., Numerous studies have indicated that PCOS is often associated with autoimmune thyroiditis. ${ }^{6,7}$ Young women with HT are most likely to present with normal thyroid function and elevated levels of thyroid peroxidase antibodies (TPOAb) and/or thyroglobulin antibodies (TGAb). ${ }^{8,9}$ Patients with PCOS in combination with HT show more severe metabolic symptoms than those with PCOS or HT alone. ${ }^{6}$

This study aims to investigate insulin secretion, insulin resistance (IR), thyroid function and the metabolic characteristics of patients with PCOS, with and without HT. 


\section{Subjects and Methods Subjects}

This study was retrospective and a total of 164 patients aged 18-35 years were diagnosed with PCOS between June 2016 and December 2018 and attended the outpatient clinic of the Department of Endocrinology at the Second Guangdong Provincial General Hospital and did not receive specific treatment. None of the women had used hormones or drugs affecting glucose metabolism in the previous 3 months.

\section{Methods}

\section{Diagnostic Criteria}

The diagnostic criteria for PCOS are those amended at the Rotterdam Conference in 2003. They are: chronic anovulation or incidental ovulation, clinical and/or biochemical signs of hyperandrogenism, and ovarian B ultrasound showing more than 12 follicles with a diameter of 2-9 $\mathrm{mm}$ in at least one ovary and/or an increase in ovarian volume of $>10 \mathrm{~mL}$. The presence of at least two of the three criteria above will confirm the diagnosis of PCOS, if other potential hyperandrogenism and ovulatory dysfunction diseases can be excluded. Patients with positive TGAb and/or TPOAb have been assumed to have Hashimoto's thyroiditis. ${ }^{9}$

\section{Measurement of Anthropometric Data}

The height and weight of the enrolled patients with PCOS were measured.

\section{Oral Glucose Tolerance Test}

After fasting for 10 hours, patients took $75 \mathrm{~g}$ of anhydrous glucose. Venous blood was drawn before $(0 \mathrm{~min})$ and at $30,60,120$, and $180 \mathrm{~min}$ after sugar ingestion in order to measure blood glucose and insulin. Blood glucose was determined by the glucose oxidase method, using an Abbott AEROSET Automatic Biochemical Analyzer, and insulin level was determined by chemiluminescence, using an Abbott AEROSET i2000.

\section{Determination of Thyroid Hormone and Thyroid Autoimmune Antibodies}

Cubital vein blood was collected after overnight fasting, and serum free triiodothyronine (FT3), serum free thyroxine (FT4), and thyroid stimulating hormone (TSH) were measured using the chemiluminescence method (Roche E601). The reference ranges for FT3 and FT4 are $3.5-5.5$ and $11.0-23.0 \mathrm{pmol} / 1$ respectively, while the reference range for TSH is $0.35-5.5 \mathrm{mIU} / 1$.

\section{Determination of Blood Lipids}

Levels of total cholesterol (TCh), triglyceride (TG), highdensity lipoprotein cholesterol (HDL-C) and low-density lipoprotein cholesterol (LDL-C) were also measured in cubital vein blood. Using an Abbott AREOSET O-ring Auto Biochemical Analyzer, TCh and TG were measured by enzymatic coupling colorimetry, while HDL-C and LDL-C were measured by the direct method. The reference ranges for $\mathrm{TCh}$, TG, HDL-C and LDL-C were 3.0-6.0 mmol/1, 0.4-1.8 mmol/1, $1.0-1.8 \mathrm{mmol} / \mathrm{l}$, and $2.0-3.1 \mathrm{mmol} / \mathrm{l}$, respectively.

\section{Statistical Analysis}

Quantitative data were expressed as mean $\pm \mathrm{SD}$, and the $t$ test was used to make comparisons between the two groups. Covariance analysis was used to correct for body mass index (BMI) in order to further analyze the differences between the two groups $(a=0.05)$. All analyses were carried out using the SPSS20.0 statistical package.

\section{Results}

Insulin levels in patients with PCOS and HT were significantly higher than in patients without HT at both 30 and 60 min, regardless of BMI correction. This difference was statistically significant. After BMI correction, there was a significant difference in fasting insulin levels between the two groups. Moreover, IR was significantly higher in the HT+ than in the HT- group after BMI correction. With respect to fat metabolism, the TCh level was significantly higher in the HT+ group after BMI correction. FT4 and FT4/TSH levels were significantly lower in the HT+ group, while the level of TSH was significantly higher, regardless of BMI correction (Tables 1 and 2).

\section{Discussion}

Our results demonstrate that, compared with patients with PCOS but no HT, patients with HT have significantly higher levels of insulin, IR, TCh and TSH. However, FT4 and FT4/TSH levels are significantly lower in the HT+ group.

Patients with PCOS often present with elevated levels of thyroid autoantibodies. Many studies over the years have shown a higher presence of autoimmune thyroiditis (AIT) in specifically polycystic ovary syndrome patients. Thyroid disorders, especially Hashimoto's thyroiditis (HT), are observed significantly more often in patients with polycystic ovary syndrome (PCOS) than in the general population - approximately $27 \%$ and $8 \%$, respectively. This is extremely important in young women, because both disorders are connected with 
Table I Comparison of Parameters Between Patients with Polycystic Ovary Syndrome with and without Hashimoto's Thyroiditis

\begin{tabular}{|c|c|c|c|}
\hline & $\begin{array}{l}\text { Hashimoto's Thyroiditis }(n=52 \text {, } \\
\text { Mean } \pm \text { SD) }\end{array}$ & $\begin{array}{l}\text { Without Hashimoto's Thyroiditis } \\
(n=|| 2, \text { Mean } \pm S D)\end{array}$ & $\mathbf{P}$ \\
\hline Age(years) & $26.22 \pm 3.93$ & $25.45 \pm 5.10$ & 0.297 \\
\hline Height(cm) & $158.18 \pm 4.95$ & $|55.86 \pm 22.0|$ & 0.464 \\
\hline Weight(kg) & $57.05 \pm 9.65$ & $61.97 \pm 16.23$ & 0.048 \\
\hline BMI(kg/m2) & $22.8 \pm 3.82$ & $24.62 \pm 6.21$ & 0.025 \\
\hline FINS(ulU/mL) & $12.47 \pm 8.03$ & $10.9 \pm 7.18$ & 0.212 \\
\hline $301 \mathrm{NS}(\mathrm{ulU} / \mathrm{mL})$ & $101.44 \pm 67.78$ & $79.78 \pm 44.23$ & 0.044 \\
\hline 60INS(ulU/mL) & II $3.78 \pm 82.72$ & $81.62 \pm 55.36$ & 0.013 \\
\hline I20INS(ulU/mL) & $87.06 \pm 87.22$ & $70.89 \pm 95.64$ & 0.302 \\
\hline I80INS(ulU/mL) & $42.21 \pm 56.99$ & $32.39 \pm 28.65$ & 0.253 \\
\hline HOMA-IR & $3.01 \pm 2.16$ & $2.69 \pm 2.21$ & 0.389 \\
\hline FT3(pmol/l) & $4.82 \pm 0.81$ & $4.94 \pm 0.56$ & 0.372 \\
\hline FT4(pmol/l) & $16.26 \pm 2.44$ & $17.54 \pm 2.65$ & 0.004 \\
\hline $\mathrm{TSH}(\mathrm{mlU} / \mathrm{l})$. & $2.82 \pm 1.23$ & $2.16 \pm 1.15$ & 0.001 \\
\hline FT4/TSH & $7.25 \pm 4.01$ & $11.05 \pm 6.87$ & $<0.001$ \\
\hline $\mathrm{HDL}(\mathrm{mmol} / \mathrm{l})$ & $1.28 \pm 0.25$ & $1.23 \pm 0.27$ & 0.298 \\
\hline $\mathrm{TG}(\mathrm{mmol} / \mathrm{l})$ & $1.31 \pm 0.89$ & $\mid .4 \pm 1.01$ & 0.582 \\
\hline $\mathrm{TC}(\mathrm{mmol} / \mathrm{l})$ & $4.69 \pm 0.98$ & $4.44 \pm 1.05$ & 0.160 \\
\hline $\mathrm{LDL}(\mathrm{mmol} / \mathrm{l})$ & $2.60 \pm 0.94$ & $2.54 \pm 0.83$ & 0.690 \\
\hline
\end{tabular}

Notes: PCOS with HT compared to PCOS without HT, patients of PCOS with HT Insulin levels at 30 and 60 minutes were significantly higher, FT4 and FT4/TSH ratios were significantly lower and TSH was significantly higher (uncorrected for BMI) $(p<0.05)$.

Table 2 Comparison of Parameters Between Patients with Polycystic Ovary Syndrome with and without Hashimoto's Thyroiditis (After Correction for Body Mass Index)

\begin{tabular}{|c|c|c|c|}
\hline & $\begin{array}{l}\text { Hashimoto's Thyroiditis }(n=52) \text {, } \\
\text { Mean }(95 \% \mathrm{Cl})\end{array}$ & $\begin{array}{l}\text { Without Hashimoto's Thyroiditis } \\
(\mathrm{n}=\mid \mathrm{I} 2), \text { Mean }(95 \% \mathrm{Cl})\end{array}$ & $\mathbf{P}$ \\
\hline FINS(ulU/mL) & |3.64(||.92, |5.35) & $10.59(9.43,11.76)$ & 0.005 \\
\hline 30INS(ulU/mL) & $103.45(88.6,118.3)$ & $78.5 \mathrm{I}(67.85,89.17)$ & 0.008 \\
\hline 60INS(ulU/mL) & $|20.5|(103.05, \mid 37.97)$ & $79.66(67.83,91.49)$ & $<0.001$ \\
\hline I20INS(ulU/mL) & $92.54(66.24,118.84)$ & $69.88(52.06,87.7)$ & 0.163 \\
\hline I80INS(ulU/mL) & $44.79(33.43,56.14)$ & $32.38(24.77,40)$ & 0.076 \\
\hline HOMA-IR & $3.33(2.8,3.85)$ & $2.62(2.26,2.97)$ & 0.030 \\
\hline FT3 (pmol/l) & $4.85(4.67,5.04)$ & $4.93(4.8,5.05)$ & 0.506 \\
\hline FT4 (pmol/l) & $16.25(15.56,16.94)$ & $17.37(16.9,17.84)$ & 0.009 \\
\hline TSH (mIU/l) & $2.79(2.46,3.13)$ & $2.16(1.94,2.39)$ & 0.002 \\
\hline FT4/TSH & $7.3(5.56,9.04)$ & $11.02(9.84,12.2)$ & 0.001 \\
\hline $\mathrm{HDL}(\mathrm{mmol} / \mathrm{l})$ & $1.26(1.19,1.33)$ & $1.23(1.18,1.28)$ & 0.542 \\
\hline $\mathrm{TG}(\mathrm{mmol} / \mathrm{l})$ & $1.4(1.15,1.66)$ & $1.37(1.19,1.55)$ & 0.835 \\
\hline $\mathrm{TC}(\mathrm{mmol} / \mathrm{l})$ & $4.73(4.44,5.02)$ & $4.37(4.17,4.58)$ & 0.049 \\
\hline $\mathrm{LDL}(\mathrm{mmol} / \mathrm{l})$ & $2.68(2.44,2.9 I)$ & $2.49(2.32,2.65)$ & 0.191 \\
\hline
\end{tabular}

Notes: Mean adjusted for BMI. PCOS with HT compared to PCOS without HT, patients of PCOS with HT Fasting insulin, 30-minute and 60-minute insulin levels were significantly higher $(\mathrm{P}<0.05)$; HOMA-IR levels were significantly higher in PCOS with HT; FT4 and FT4/TSH ratios were significantly lower and TSH was significantly higher; TC was significantly higher (after correction for BMI) $(\mathrm{P}<0.05)$.

fertility problems. As HT and PCOS occur together, fertility problems may become a serious clinical issue in these patients. ${ }^{7,10,11}$ The first prospective study was completed by Janssen et al. ${ }^{11}$ The results showed that levels of TPOAb and TGAb, indicative of HT, were increased in the PCOS group compared with the control group. Although all subjects had thyroid hormone levels in the normal range, the mean TSH level in the PCOS group was significantly higher than in the control group. In addition, a TSH level above the upper limit of normal was seen more often in the PCOS group. TPOAb 
and TGAb were also significantly increased in the PCOS group.

An Iranian case-control study found that the level of TPOAb in patients with PCOS was significantly higher than that in controls. However, there was no difference in serum TSH and TGAb levels between the PCOS group and the control group. ${ }^{12} \mathrm{~A}$ recent study in Italy suggests that the prevalence of HT in patients with PCOS is significantly higher than in controls. ${ }^{13}$

Furthermore, Arduc et $\mathrm{al}^{14}$ confirm that HT occurs more frequently in patients with PCOS. In this study, $\mathrm{TSH}, \mathrm{TPOAb}$ and TGAb levels of patients with PCOS were significantly higher than those of the control group. However, there were differences in FT3, FT4 and thyroid volume between the two groups. The slightly higher percentage of patients with HT in this study, compared with other studies, is likely due to different diagnostic criteria.

Mueller ${ }^{15}$ reports that even when TSH concentration is in the normal range, BMI and IR are higher in women with PCOS whose TSH level is above $2 \mathrm{mIU} / 1$ compared with those with a TSH level below $2 \mathrm{mIU} / \mathrm{l}$. Furthermore, IR is independent of BMI and age. This study suggests that a TSH value of about $2 \mathrm{mIU} / 1$ has optimum sensitivity and specificity to identify IR in women with PCOS.

Thyroid function appears to affect both the clinical and biochemical parameters of PCOS. ${ }^{6,16,17}$ In cases of hypothyroidism, or when the TSH is in the upper limit of the normal reference range, PCOS combined with HT produces more significant metabolic changes compared with HT or PCOS alone. In fact, girls with HT and PCOS have a higher BMI, higher fasting glucose and IR, and higher cholesterol than those with HT alone or controls. ${ }^{18}$ Similarly, women with PCOS with subclinical hypothyroidism also have higher $\mathrm{TG}$ and fasting insulin levels and higher IR than those with normal thyroid function. However, TC, HDL-C, and LDL-C show no differences. $^{19,20}$

The findings of our study suggest that IR is more marked in PCOS with HT than in PCOS alone. While the TCh level was significantly higher in the HT+ group, blood glucose levels did not differ between the two groups at any time point during the oral glucose tolerance test. This suggests that patients with PCOS and HT have abnormal lipid metabolism compared with patients with PCOS alone. However, the weight of patients with PCOS and HT was significantly lower than that of patients with PCOS alone. The reason for this is unclear, although it may be due to the fact that almost all the HT patients enrolled in this study had normal thyroid function (only 3 patients had subclinical hypothyroidism and TSH $<10 \mathrm{mIU} / \mathrm{l})$.

\section{Conclusion}

This comparative study of patients with PCOS with and without HT found that insulin levels at 30 and $60 \mathrm{~min}$ are significantly higher in patients with PCOS and HT than in those without HT. This difference remained significant after correction for BMI. Furthermore, fasting insulin levels were also statistically different between the two groups. IR and TCh levels were also significantly higher in the HT+ group after BMI correction. Testing for thyroid function parameters revealed that FT4 and FT4/TSH levels in the HT+ group were significantly lower than in the HTgroup, regardless of BMI correction, while the TSH level was significantly higher.

These differences suggest that HT may aggravate IR and result in relatively low thyroid function in patients with PCOS. Thus, thyroid function and autoimmune status in patients with PCOS should be evaluated in clinical practice.

\section{Ethics Approval and Consent to Participate}

This study was conducted in accordance with the Declaration of Helsinki. This study was conducted with approval from the Ethics Committee of Guangdong Second Provincial General Hospital. Written informed consent was obtained from all participants.

\section{Funding}

Subjects: A study of the mechanism of ovarian syndrome with autoimmune thyroid disease. Guangdong Provincial Science and Technology Department Research Fund Project (2017ZC0284).

\section{Disclosure}

The authors report no conflicts of interest in this work.

\section{References}

1. Rotterdam ESHRE/ASRM-Sponsored PCOS consensus workshop group; Revised 2003 consensus on diagnostic criteria and long-term health risks related to polycystic ovary syndrome (PCOS). Hum Reprod. 2004;19(1):41-47. doi:10.1093/humrep/deh098

2. Azziz R. Polycystic Ovary Syndrome. Obstet Gynecol. 2018;132 (2):321-336. doi:10.1097/AOG.0000000000002698

3. Trikudanathan S. Polycystic ovarian syndrome. Med Clin North Am. 2015;99(1):221-235. doi:10.1016/j.mena.2014.09.003

4. Saei Ghare Naz M, Rostami Dovom M, Ramezani Tehrani F. The menstrual disturbances in endocrine disorders: a narrative review. Int J Endocrinol Metab. 2020;18(4):e106694. doi:10.5812/ijem.106694 
5. Unuane D, Velkeniers B. Impact of thyroid disease on fertility and assisted conception. Best Pract Res Clin Endocrinol Metab. 2020;34 (4):101378. doi:10.1016/j.beem.2020.101378

6. Gaberšček S, Zaletel K, Schwetz V, Pieber T, Obermayer-Pietsch B, Lerchbaum E. Mechanisms in endocrinology: thyroid and polycystic ovary syndrome. Eur J Endocrinol. 2015;172(1):R9-R21. doi:10.1530/ EJE-14-0295

7. Kowalczyk K, Franik G, Kowalczyk D, Pluta D, Blukacz Ł, Madej P. Thyroid disorders in polycystic ovary syndrome. Eur Rev Med Pharmacol Sci. 2017;21(2):346-360.

8. Hollowell JG, Staehling NW, Flanders WD, et al. T(4), and thyroid antibodies in the United States population (1988 to 1994): national Health and Nutrition Examination Survey (NHANES III). J Clin Endocrinol Metab. 2002;87(2):489-499.

9. Spencer CA, Hollowell JG, Kazarosyan M, Braverman LE. National Health and Nutrition Examination Survey III thyroid-stimulating hormone (TSH)-thyroperoxidase antibody relationships demonstrate that TSH upper reference limits may be skewed by occult thyroid dysfunction. $J$ Clin Endocrinol Metab. 2007;92(11):4236-4240. doi:10.1210/jc.2007-0287

10. Romitti M, Fabris VC, Ziegelmann PK, Maia AL, Spritzer PM. Association between PCOS and autoimmune thyroid disease: a systematic review and meta-analysis. Endocr Connect. 2018;7 (11):1158-1167. doi:10.1530/EC-18-0309

11. Janssen OE, Mehlmauer N, Hahn S, Offner AH, Gärtner R. High prevalence of autoimmune thyroiditis in patients with polycystic ovary syndrome. Eur J Endocrinol. 2004;150(3):363-369. doi:10.1530/ eje. 0.1500363

12. Kachuei M, Jafari F, Kachuei A, Keshteli AH. Prevalence of autoimmune thyroiditis in patients with polycystic ovary syndrome. Arch Gynecol Obstet. 2012;285(3):853-856. doi:10.1007/s00404-011-2040-5

13. Garelli S, Masiero S, Plebani M, et al. High prevalence of chronic thyroiditis in patients with polycystic ovary syndrome. Eur J Obstet Gynecol Reprod Biol. 2013;169(2):248-251. doi:10.1016/j. ejogrb.2013.03.003
14. Arduc A, Aycicek Dogan B, Bilmez S, et al. High prevalence of Hashimoto's thyroiditis in patients with polycystic ovary syndrome: does the imbalance between estradiol and progesterone play a role? Endocr Res. 2015;40(4):204-210. doi:10.3109/ 07435800.2015 .1015730

15. Mueller A, Schöfl C, Dittrich R, et al. Thyroid-stimulating hormone is associated with insulin resistance independently of body mass index and age in women with polycystic ovary syndrome. Hum Reprod. 2009;24(11):2924-2930. doi:10.1093/humrep/ $\operatorname{dep} 285$

16. Singh J, Wong H, Ahluwalia N, Go RM, Guerrero-Go MA. Metabolic, Hormonal, Immunologic, and genetic factors associated with the incidence of thyroid disorders in polycystic ovarian syndrome patients. Cureus. 2020;12(11):e11681.

17. Ulrich J, Goerges J, Keck C, Müller-Wieland D, Diederich S, Janssen OE. Impact of autoimmune thyroiditis on reproductive and metabolic parameters in patients with polycystic ovary syndrome. Exp Clin Endocrinol Diabetes. 2018;126(4):198-204. doi:10.1055/s-0043-110480

18. Ganie MA, Marwaha RK, Aggarwal R, Singh S. High prevalence of polycystic ovary syndrome characteristics in girls with euthyroid chronic lymphocytic thyroiditis: a case-control study. Eur J Endocrinol. 2010;162(6):1117-1122. doi:10.1530/EJE-091012

19. Celik C, Abali R, Tasdemir N, et al. Is subclinical hypothyroidism contributing dyslipidemia and insulin resistance in women with polycystic ovary syndrome? Gynecol Endocrinol. 2012;28(8):615-618. doi:10.3109/09513590.2011.650765

20. Fatima M, Amjad S, Sharaf Ali H Sr, et al. Correlation of Subclinical Hypothyroidism With Polycystic Ovary Syndrome (PCOS). Cureus. 2020;12(5).

\section{Publish your work in this journal}

Diabetes, Metabolic Syndrome and Obesity: Targets and Therapy is an international, peer-reviewed open-access journal committed to the rapid publication of the latest laboratory and clinical findings in the fields of diabetes, metabolic syndrome and obesity research. Original research, review, case reports, hypothesis formation, expert opinion and commentaries are all considered for publication. The manuscript management system is completely online and includes a very quick and fair peer-review system, which is all easy to use. Visit http://www.dovepress.com/testimonials.php to read real quotes from published authors. 